\title{
COSTAL BATHYMETRY ESTIMATION FROM MULTISPECTRAL IMAGE WITH BACK PROPAGATION NEURAL NETWORK
}

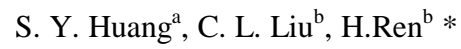 \\ ${ }^{a}$ Dept. of Computer and Information Engineering, National Central University, Taiwan - syas1983@ gmail.com \\ ${ }^{\mathrm{b}}$ Center for Space and Remote Sensing Research, National Central University, Taiwan - (ericleo, hren)@ csrsr.ncu.edu.tw
}

Commission VIII, WG VIII/9

KEY WORDS: Bathymetry, Multispectral Image, Stereo Image, Back Propagation Neural Network

\begin{abstract}
:
Bathymetric data in coastal area are important for marine sciences, hydrological applications and even for transportation and military purposes. Compare to traditional sonar and recent airborne bathymetry LIDAR systems, optical satellite images can provide information to survey a large area with single or multiple satellite images efficiently and economically. And it is especially suitable for coastal area because the penetration of visible light in water merely reaches 30 meters. In this study, a three-layer back propagation neural network is proposed to estimate bathymetry. In the learning stage, some training samples with known depth are adopted to train the weights of the neural network until the stopping criterion is satisfied. The spectral information is sent to the input layer and fits the true water depth with the output. The depths of training samples are manually measured from stereo images of the submerged reefs after water refraction correction. In the testing stage, all non-land pixels are processed. The experiments show the mean square errors are less than 3 meters.
\end{abstract}

\section{INTRODUCTION}

Bathymetric data in coastal area are important for marine sciences, hydrological applications and even for transportation and military purposes. The traditional approach relies on sonar system which can measure depth more than hundreds of meters (Baban, 1993). However, it encounters two limitations. First, sonar is not suitable for shallow water area because the ship usually with draft more than five meters may be damaged in shallow area. Secondly, ship sonar can only measure the depth with limited area along the track and it needs to sail back and forth to cover the area. The recent technology of airborne bathymetry LIDAR can overcome the first drawback, but it still cannot survey a large area in short time. Also the operational cost is very high. Bathymetry estimation from optical satellite images has drawn a lot of attention recently (Benny, 1983). It is efficient and economic since it can survey a large area with single or multiple satellite images. It is especially suitable for coastal area because the penetration of visible light in water merely reaches 30 meters.

The spectral information collected from multispectral image depends on three factors: water depth, water absorption coefficient and bottom materials. Since the relationship among these three factors is nonlinear (Lyzenga, 1978), supervised neural networks are considered. In this study, a three-layer back propagation neural network (Haykin, 1998) is proposed to estimate bathymetry. In the learning stage, some training samples with known depth are adopted to train the weights of the neural network until the stopping criterion is satisfied. The spectral information is sent to the input layer and fits the true water depth with the output. The depths of training samples are manually measured from stereo images of the submerged reefs after water refraction correction. In the testing stage, all non-land pixels are processed. The experimental results show the mean square errors in training stage and testing stage are 2.5 and 2.7 meters respectively. And the trained neural network can also applied to another image scene with mean square error less than 3 meters.

\section{BACK PROPAGATION NEURAL NETWORK}

Back propagation neural network is a traditional artificial neural network. It simulates the neuron learning from feedback. The structure of back propagation neural network contain one input layer, one output layer and one or more hidden layers (Fig 1). And neurons between adjacent layers are connected with weights which will be adjusted in training stage. The input of each neuron is the weighted sum of all outputs from previous layer, and a sigmoid function is applied to calculate the output (Fig 2).

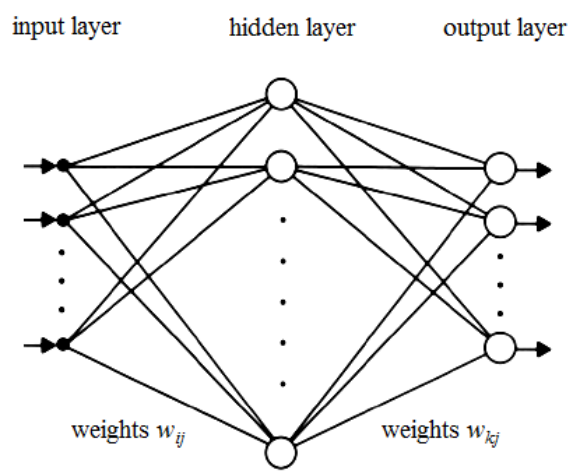

Figure 1. Three-layer neural network structure

\footnotetext{
* Corresponding author
} 


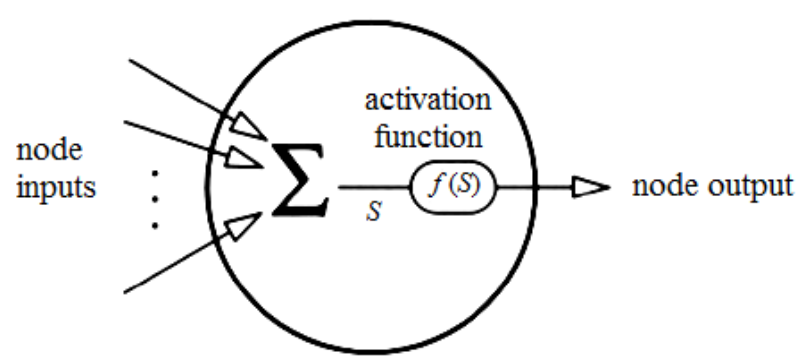

Figure 2. The components of a neuron

This network has two stages: training stage and testing stage. Each iteration of the training stage, training samples are sent to input layer, and the differences of outputs and desired outputs are back propagated to update the weights. The training stage is terminated when the differences are smaller than a preset threshold or the maximum iteration is reached. After the training stage, the whole data set will be processed in testing stage.

\section{EXPERIMENTAL RESULT}

The WorldView-2 image scene around Jing-Hong Island in South China Sea is selected for experiment as shown in Figure 3. There are 563 ground truth samples indicated by yellow circles. The true water depth is measured manually from stereo images. Among those samples, 100 of them are randomly selected for training. The digital numbers of all spectral bands are the input to the three-layer back propagation neural network and the geometric height is the desired output. After stopping criterion is met, the output and ground truth of training samples are shown in Figure 4 (a), and the Root Mean Squares Error (RMSE) of training samples is 2.5 meters. Expect some deep samples are under estimated, most of the samples are well fitted. The rest 463 samples are for testing shown in Figure 4 (b), and the RMSE of training samples is 2.7 meters. Finally, a $6000 \times 6000$ sub-image from bottom left island is cropped for processing (Figure 5). The 2D and 3D view of bathymetry in geometric height are shown in Figure 6 (a) and (b).

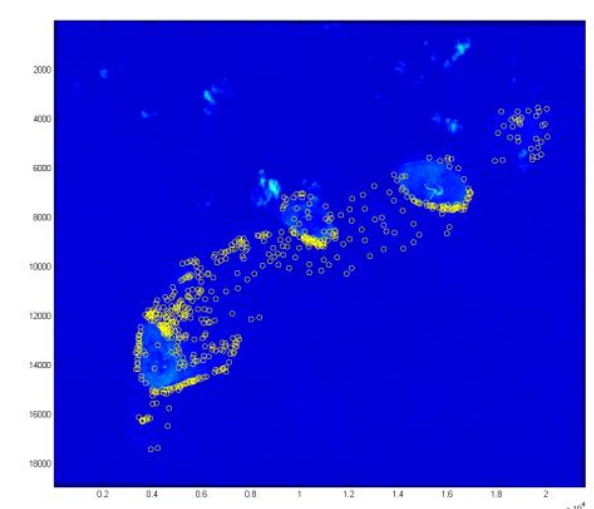

Figure 3. WorldView-2 image around Jing-Hong Island with ground truth position

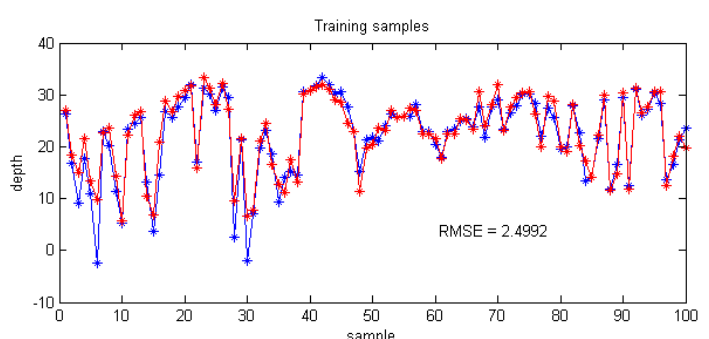

(a)

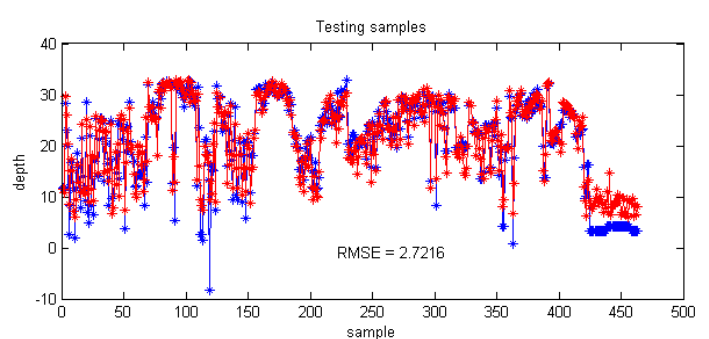

(b)

Figure 4. Error assessment of training and testing samples for back propagation neural network

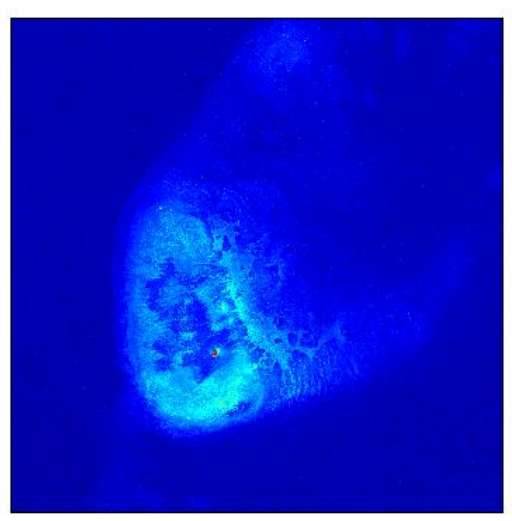

Figure 5. The sub-image scene from WorldView-2

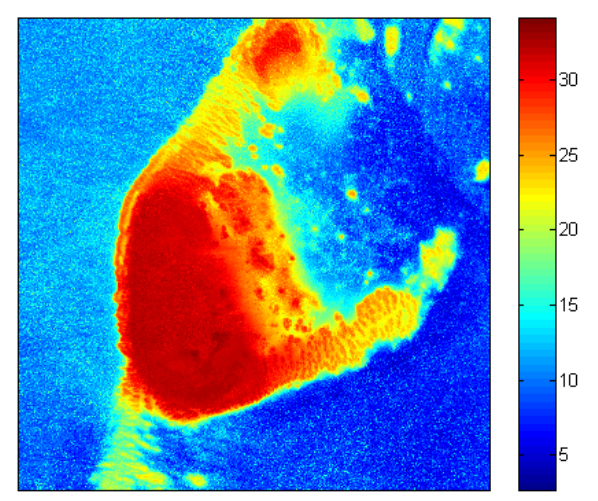

(a) 


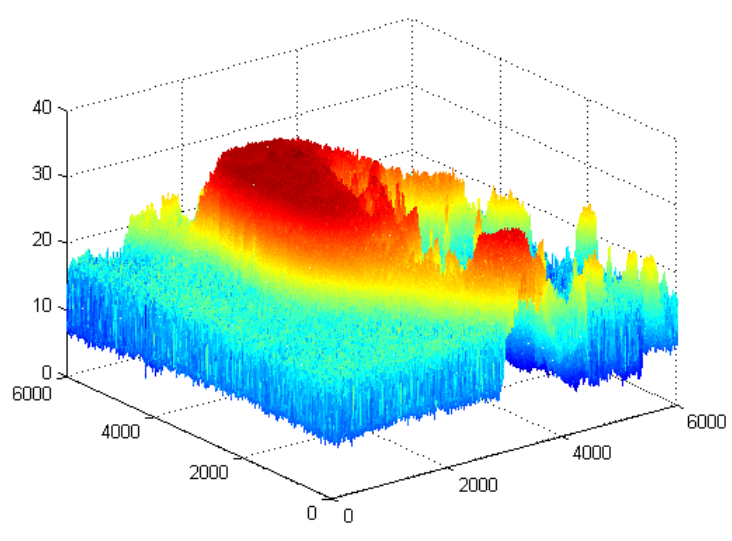

(b)

Figure 6. The bathymetry estimation in $2 \mathrm{D}$ and $3 \mathrm{D}$

\section{CONCLUSION}

Because of the penetration of visible light in water and the large swath, optical satellite images are suitable for water depth estimation in shallow water area, especially for islands which cannot be easily reached. In this study, a back propagation neural network is adopted for this purpose. Our preliminary result for WorldView-2 multispectral remote sensing images has RMSE less than 3 meters for both training and testing samples. Although we do not have ground truth for the whole area, the reconstructed bathymetry in $2 \mathrm{D}$ and $3 \mathrm{D}$ is reasonable. Finally, since the error assessment of training samples indicates the under estimation in deep area, the improvements can be expected with some modifications.

\section{REFERENCES}

Baban, S.M.J., 1993, The evaluation of different algorithms for bathymetry charting of lakes using Landsat imagery. International Journal of Remote Sensing, 14, pp. 22632273.

Benny, A.H. and Dawson, G.J., 1983, Satellite imagery as an aid to bathymetry charting in the Red Sea. The Cartographic Journal, 20, pp. 5-16.

Haykin, S., 1998, Neural Networks - A Comprehensive Fundation, 2nd edition, Prentice Hall.

Lyzenga, D.R., 1978, Passive remote sensing techniques for mapping water depth and bottom features. Applied Optics, 17, pp. 379-383. 